EXTENDED REPORT

\title{
ANCA associated pauci-immune retinal vasculitis
}

\author{
M J Gallagher, K G-J Ooi, M Thomas, M Gavin
}

Br J Ophthalmol 2005;89:608-611. doi: 10.1136/bjo.2004.051177

Background: Antinuclear cytoplasmic antibodies (ANCA) are useful diagnostic serological markers for the most common forms of necrotising vasculitis. ANCA associated vasculitides represent distinctive clinicopathological categories-for example, Wegener's granulomatosis, Churg-Strauss syndrome, microscopic polyangiitis, and idiopathic necrotising crescentic glomerulonephritis, collectively known as the small vessel pauci-immune vasculitides.

See end of article for authors' affiliations

\section{Correspondence to:}

Correspondence to:
Dr M J Gallagher, Tennent Institute of Ophthalmology, Gartnavel General

Hospital, Glasgow G12 9TA, UK; migallagher@ doctors.org.uk

Accepted for publication 6 September 2004
Method: Three cases of ANCA associated pauci-immune retinal vasculitis are described. Their systemic features are described and the clinical significance of ANCA as a diagnostic test in relation to retinal vasculitis discussed.

Results: These three cases represent a spectrum of clinical features associated with retinal vasculitis. Two cases have evolved into clinical recognisable entities as microscopic polyangiitis. Adherence to the international consensus statement on testing and reporting of ANCA is recommended and the authors speculate that the incidence of microscopic polyangiitis may be underestimated because of the underrecognition of systemic involvement in patients with retinal vasculitis.

Conclusion: The receipt of a positive ANCA result should always raise the suspicion of a pauci-immune systemic vasculitis and prompt appropriate investigation. The authors emphasise the importance of the evaluation of systemic features in these patients with retinal vasculitis, enabling earlier recognition and thereby preventing significant morbidity and mortality.
A ntineutrophil cytoplasmic autoantibodies (ANCA) are useful diagnostic serological markers for the most common forms of necrotising vasculitis providing a means of categorising vasculitides so that diagnostically shared pathological and clinical characteristics can be correlated.

ANCA produce two immunostaining patterns on alcohol fixed neutrophils: a cytoplasmic (C-ANCA) and a perinuclear (P-ANCA) pattern. P-ANCA's main specificity is myeloperoxidase (MPO). ${ }^{1}$ C-ANCA is mainly produced by antibodies against PR3, a third serine protease from azurophilic myeloid granules. ${ }^{2}$ The international consensus statement on testing and reporting of ANCA was developed to minimise the technical difficulties of ANCA testing and its interpretation. ${ }^{3}$

It should be noted that systemic vasculitis may occur in the absence of a positive ANCA and, conversely, positive ANCAs may be found in the absence of vasculitis-for example, with infection and neoplasia. Receipt of a positive ANCA result should always raise the suspicion of a pauci-immune systemic vasculitis and prompt appropriate investigation.

\section{CASE REPORTS}

\section{Case 1}

A 35 year old woman presented with pain and blurring of vision in her right eye. She related systemic complaints of malaise, lethargy, weight loss, and intermittent night sweats. Blood pressure was normal, urinalysis demonstrating moderate haem ++ .

Examination demonstrated a right visual acuity of 3/60 (improving to 6/9 with pinhole). There were cells+ in the anterior chamber, the posterior segment demonstrating vitreous cells+++. Funduscopy revealed peripheral vascular sheathing (fig 1).

Blood results revealed a positive P-ANCA on immunofluorescence (MPO/PR3 testing not available at this time) with an elevated erythrocyte sedimentation rate $(94 \mathrm{~mm}$ in the first hour), and C reactive protein $(20 \mathrm{mg} / \mathrm{l})$. Serum antinuclear antibody testing was intermittently positive at

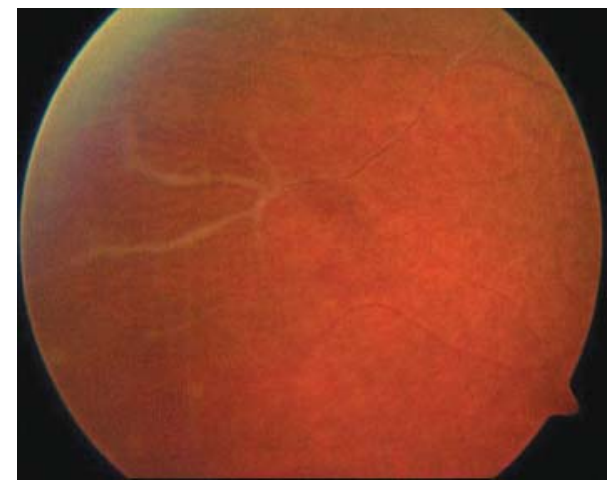

Figure 1 Peripheral retinal vasculitis with vascular sheathing

1:40 (on Hep2000 cells). Investigations for retinal vasculitisnamely, full blood count, urea and electrolytes, liver function tests, serum angiotensin converting enzyme, complement, rheumatoid factor, HLA B-27, serum VDRL, anti-cardiolipin antibodies, and a chest radiograph were normal.

The patient was treated conservatively with topical corticosteroids. Further exacerbations, resulted in bilateral ocular involvement with extensive peripheral retinal vasculitis (fig 2). Symptoms included fatigue, lethargy, arthralgia, weight loss, and loin pain. Systemic examination demonstrated finger pulp haemorrhages indicative of cutaneous vasculitis.

Renal ultrasound was normal. Repeat urinalysis demonstrated persistent haematuria. Serial mid-stream urine specimens were negative demonstrating no evidence of pus cells or organisms. A renal biopsy demonstrated an interstitial lymphocytic infiltrate with absence of immune complex deposition indicative of glomerulonephritis (fig 3). Renal

Abbreviations: ANA, antinuclear antibody; ANCA, antinuclear cytoplasmic antibodies; MPO, myeloperoxidase 


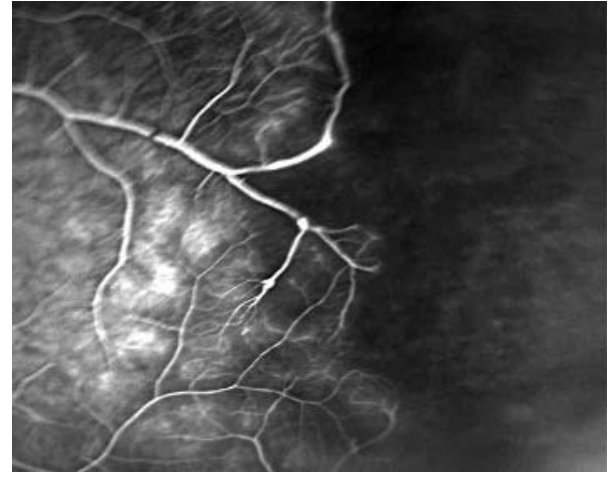

Figure 2 Fundal fluorescein angiography demonstrating extensive peripheral retinal vasculitis.

function was normal. P-ANCA was persistently positive but was negative for MPO and PR3 antibodies. Serum antinuclear antibody (ANA) was intermittently positive (low titre). Serum ANA, however, was negative when serum ANCA was at its strongest titre $(1: 640)$ suggesting that she did indeed have a positive ANCA (fig 4).

The patient responded favourably to systemic steroid treatment with adjunctive cyclosporin (150 mg twice daily). Further monitoring demonstrated a decline in renal function with an isotope glomerular filtration rate of $63 \mathrm{ml}$ per minute with normal serum urea and creatinine levels. Cyclosporin therapy was discontinued and mycophenolate mofetil ( $1 \mathrm{~g}$ twice daily) commenced to continue immunosuppression which has protected against further renal compromise.

\section{Case 2}

A 39 year old woman presented with a painless reduction of vision in her left eye. She related paraesthesia in both her legs over the previous few months. Blood pressure was 160/ $100 \mathrm{~mm} \mathrm{Hg}$, with normal urinalysis.

Examination of her left posterior segment demonstrated a vitreal haemorrhage with neovascularisation in the left superotemporal quadrant. The right posterior segment also demonstrated active retinal vasculitis. A vasculitic screen was performed.

Serum P-ANCA was positive with a 1:20 titre. Anti-MPO and PR3 antibodies were negative. Serum ANA was negative. Routine bloods were normal. C reactive protein was mildly elevated at $21 \mathrm{mg} / \mathrm{l}$, with a normal erythrocyte sedimentation rate. Computed tomography (CT), brain, and electroencephalogram (EEG) measurements were normal. There were no other neurological symptoms to suggest multiple sclerosis. A clinical diagnosis of presumed mononeuritis multiplex was made with no cause elucidated.

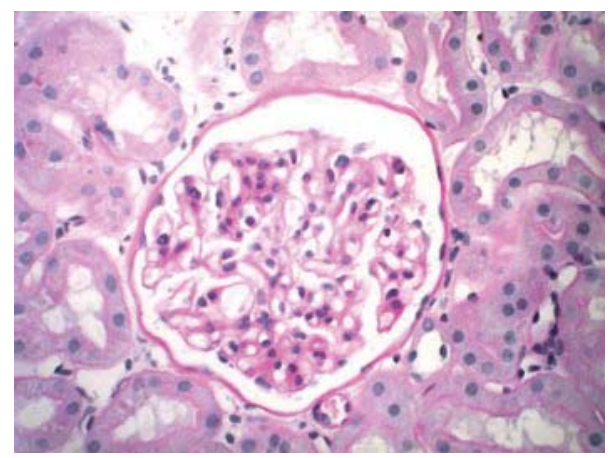

Figure 3 Renal biopsy demonstrating interstitial lymphocytic infiltration and paucity of immune complex deposition.

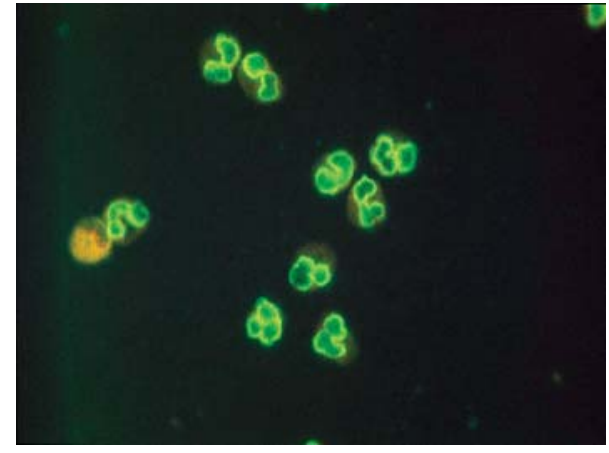

Figure 4 Slide demonstrating immunostaining positivity for serum ANCA.

She underwent a sectoral panretinal photocoagulation and was commenced on cyclosporin therapy ( $100 \mathrm{mg}$ twice daily) to which she responded well with quiescence of her retinal vasculitis.

\section{Case 3}

A 28 year old woman presented with a reduction in vision in her left eye. Blood pressure was 115/84 mm Hg with normal urinalysis.

Examination demonstrated a left visual acuity of hand movements, a quiet anterior chamber with evidence of posterior segment vitreal haemorrhage. On resolution of the vitreal haemorrhage over a 7 week period, further reexamination demonstrated vitreal cells++ with peripheral retinal vasculitis and a localised area of neovascularisation inferiorly. Investigation of this patient's retinal vasculitis demonstrated a positive P-ANCA (1:160) on immunofluorescence, which was negative for anti-MPO and PR3 antibodies. Serum ANA was negative. Routine bloods and renal function were normal.

This patient presented with pauci-immune retinal vasculitis without systemic involvement. She was commenced on cyclosporin (125 mg twice daily) (patient was reluctant to commence steroid treatment) and responded well, with quiescence of the vasculitis, an improvement in visual acuity to 6/9 left eye, with no further episodes of vitreal haemorrhage.

\section{DISCUSSION}

These cases represent a spectrum of clinical features associated with retinal vasculitis. The first case presented with a chronic history of arthralgia, followed by systemic features of fatigue, lethargy, weight loss, mononeuritis multiplex, finger pulp haemorrhages, and loin pain.

Our patient developed a progressive deterioration in glomerular filtration rate, indicative of intrinsic renal disease. No immune complex deposition was seen on renal biopsy. In conjunction with a positive P-ANCA and in the absence of granulomatous inflammation or the presence of eosinoplilia or asthma in this patient, a diagnosis of microscopic polyangiitis was made.

It is highly probable that our second case is also a case of evolving MPA (according to the international consensus statement) presenting with bilateral retinal vasculitis and peripheral neuropathy in association with a positive P-ANCA. Our third P-ANCA positive retinal vasculitis case continues to be screened for associated systemic features. Our paper describes three cases of retinal vasculitis, all with positive serum P-ANCA, two of which have evolved into clinically recognisable entities as MPA. Jennette et al suggest that patients who initially have clinical manifestations consistent 
Table 1 Serum ANCA specificity in untreated disease

\begin{tabular}{lllr}
\hline & PR3-ANCA & MPO-ANCA & Negative \\
\hline Microscopic polyangiitis & $40 \%$ & $50 \%$ & $10 \%$ \\
Wegener's granulomatosis & $75 \%$ & $20 \%$ & $5 \%$ \\
Churg-Strauss syndrome & $10 \%$ & $60 \%$ & $30 \%$ \\
\hline Adapted from Jennette et al. & & & \\
\hline
\end{tabular}

with MPA may subsequently develop features of Wegener's granulomatosis. ${ }^{4}$

These patients presented with retinal vasculitis in association with a positive P-ANCA on immunofluorescence, negative for anti-MPO and PR3 antibodies. Despite the absence of either MPO or PR3 antibodies two of our patients showed evidence of systemic vasculitic involvement. We suggest that any patient with P-ANCA positivity (by ELISA or indirect immunofluorescence) and retinal vasculitis, regardless of specificity should be reviewed for evidence of systemic vasculitis. When systemic clinical features are present the demonstration of ANCA is probably 95\% sensitive and 90\% specific for these diseases and has a much higher positive predictive value than in other hospitalised patients. ${ }^{5}$

In an attempt to clarify the testing and reporting of ANCA serology, an international consensus statement was formulated. The consensus recommends testing for ANCA in patients with:

- glomerulonephritis

- pulmonary haemorrhage

- cutaneous vasculitis with systemic features

- mononeuritis multiplex or other peripheral neuropathy

- longstanding sinusitis or otitis

- subglottic tracheal stenosis

- retro-orbital mass.

The presence of one of these clinical features, or other strong clinical evidence for small vessel vasculitis with a positive ANCA test provides $90 \%$ confirmation of pauciimmune small vessel vasculitides. ${ }^{3}$ The antigen specificity of ANCA points towards different clinicopathological phenotypes of the small vessel pauci-immune vasculitides. A negative result does not rule out this category of vasculitides (table 1). ${ }^{4}$

Microscopic polyangiitis is a now recognised pauciimmune systemic vasculitis. Small vessel involvement is the definitive diagnostic criterion of MPA and excludes the diagnosis of polyarteritis nodosa even if medium sized

Table 2 Clinical manifestations of microscopic polyangiitis

\begin{tabular}{ll}
\hline Renal manifestations & $78.8 \%$ \\
Weight loss & $72.9 \%$ \\
Skin involvement & $62.4 \%$ \\
Fever & $53.3 \%$ \\
Mononeuritis multiplex & $57.6 \%$ \\
Athralgia & $50.6 \%$ \\
Myalgia & $48.2 \%$ \\
Hypertension & $34.1 \%$ \\
Gastrointestinal involvement & $30.6 \%$ \\
Pulmonary involvement & $24.7 \%$ \\
Central nervous system Involvement & $11.8 \%$ \\
Digital ischaemia & $7.1 \%$ \\
Ocular involvement & $1.2 \%$ \\
\hline & \\
\hline \multirow{2}{*}{ Percentages reflecting the frequency of involvement in a study group of } \\
85 patients positive for microscopic polyangiitis. Adapted from Guillevin \\
et al. ${ }^{\circ}$
\end{tabular}

arterial lesions are also seen. MPA has an incidence of approximately 1:100 000 with a slight female predominance, a mean age of onset of 50 years, although any age may be affected. ${ }^{4}$ Guillevin et $a l^{6}$ have described the clinical features in 85 patients (table 2).

The pathophysiological relevance of ANCA has been studied extensively. It has been demonstrated ${ }^{7}$ that IgG fractions from ANCA positive sera, are capable of inducing tumour necrosis factor primed neutrophils to release lysosomal enzymes and produce reactive oxygen species inducing vascular injury. ANCA associated vasculitides are described as being pauci-immune, characterised by necrotising inflammation but with a paucity of immune deposits. ${ }^{8}$

The recommended treatment for MPA is high dose corticosteroids and cyclophosphamide. 'Dandekar et al, in their recent series on ocular involvement in systemic vasculitis, relate that treatment with immunosuppressants resulted in a reduction in P-ANCA titre with dramatic improvement of symptoms. ${ }^{10}$

In conclusion, we advocate the testing of all patients with retinal vasculitis for serum ANCA. We acknowledge that ANCA specificity does not allow us to distinguish diverse forms of necrotising vasculitis. We speculate that the incidence of MPA may be underestimated because of the under-recognition of systemic involvement in patients with retinal vasculitis. We emphasise the importance in this regard of recognition of these clinical features and the differential diagnosis of these pauci-immune small vessel vasculitides so that prompt immunosuppressive therapy is instituted and patient morbidity, if not mortality, is prevented.

\section{ACKNOWLEDGEMENTS}

We thank the library staff and Medical Illustration Department at Gartnavel General Hospital, Glasgow.

\section{Authors' affiliations}

M J Gallagher, K Ooi, M Gavin, Tennent Institute of Ophthalmology, Gartnavel General Hospital, Glasgow, UK

M Thomas, Department of Immunology, Western Infirmary, Glasgow GI1, UK

\section{REFERENCES}

1 Falk RJ, Jennette JC. Antineutrophil cytoplasmic antibodies with specificity for myeloperoxidase in patients with systemic vasculitis and idiopathic necrotising and crescentic glomerulonephritis. N Engl J Med 1998;318:1651-7.

2 Niles JL, McCluskey RT, Ahmed MF, et al. Wegener's granulomatosis autoantigen is a novel neutrophil serine proteinase. Blood 1989;74:1888-93.

3 Savige J, Gillis D, Davies D, et al. International consensus statement on testing and reporting of antineutrophil cytoplasmic antibodies (ANCA). Am J Clin Pathol 1999;111:507-13.

4 Jennette JC, Thomas BD, Falk RJ. Microscopic Polyangiitis. Sem Diagn Pathol 2001;18:1:3-13.

5 Jennette JC. Antineutrophil cytoplasmic antibody associated diseases; a pathologist's perspective. Am J Kidney Dis 1991;28:164-70.

6 Guillevin L, Durand-Gasselin B, Cevallos R, et al. Microscopic polyangiitis: clinical and laboratory findings in eighty-five patients. Arthritis Rheum 1999;42:3:421-30.

7 Falk R, Terrell R, Charles L, et al. Antineutrophil cytoplasmic antibodies induce neutrophils to degranulate and produce oxygen radicals in vitro. Proc Natl Acad Sci USA 1990;87:4115-19.

8 Harper L, Savage CO. Pathogenesis of ANCA-associated systemic vasculitis. J Pathol 2000;190:349-59. 
9 Guillevin L, Lhote F. Distinguishing polyarteritis nodosa from microscopic polyangiitis and implications for treatment. Curr Opin Rheumatol 1995;7:20-24
10 Dandekar SS, Narendran NN, Edmunds B, et al. Ocular involvement in systemic vasculitis associated with perinuclear antineutrophil cytoplasmic antibodies. Arch Ophthalmol 2004;122:786-7.

\section{Clinical Evidence-Call for contributors}

Clinical Evidence is a regularly updated evidence-based journal available worldwide both as a paper version and on the internet. Clinical Evidence needs to recruit a number of new contributors. Contributors are healthcare professionals or epidemiologists with experience in evidence-based medicine and the ability to write in a concise and structured way.

Areas for which we are currently seeking authors:

- Child health: nocturnal enuresis

- Eye disorders: bacterial conjunctivitis

- Male health: prostate cancer (metastatic)

- Women's health: pre-menstrual syndrome; pyelonephritis in non-pregnant women

However, we are always looking for others, so do not let this list discourage you.

Being a contributor involves:

- Selecting from a validated, screened search (performed by in-house Information Specialists) epidemiologically sound studies for inclusion.

- Documenting your decisions about which studies to include on an inclusion and exclusion form, which we keep on file.

- Writing the text to a highly structured template (about 1500-3000 words), using evidence from the final studies chosen, within 8-10 weeks of receiving the literature search.

- Working with Clinical Evidence editors to ensure that the final text meets epidemiological and style standards.

- Updating the text every six months using any new, sound evidence that becomes available. The Clinical Evidence in-house team will conduct the searches for contributors; your task is simply to filter out high quality studies and incorporate them in the existing text.

- To expand the topic to include a new question about once every 12-18 months.

If you would like to become a contributor for Clinical Evidence or require more information about what this involves please send your contact details and a copy of your CV, clearly stating the clinical area you are interested in, to Klara Brunnhuber (kbrunnhuber@ bmigroup.com).

\section{Call for peer reviewers}

Clinical Evidence also needs to recruit a number of new peer reviewers specifically with an interest in the clinical areas stated above, and also others related to general practice. Peer reviewers are healthcare professionals or epidemiologists with experience in evidence-based medicine. As a peer reviewer you would be asked for your views on the clinical relevance, validity, and accessibility of specific topics within the journal, and their usefulness to the intended audience (international generalists and healthcare professionals, possibly with limited statistical knowledge). Topics are usually 1500-3000 words in length and we would ask you to review between 2-5 topics per year. The peer review process takes place throughout the year, and our turnaround time for each review is ideally 10-14 days.

If you are interested in becoming a peer reviewer for Clinical Evidence, please complete the peer review questionnaire at www.clinicalevidence.com or contact Klara Brunnhuber (kbrunnhuber@bmigroup.com). 